J. Clin. Chem. Clin. Biochem.

Vol. 17, 1979, pp. 35-43

\title{
Simultanbestimmung von Antiepileptika in klinischen Laboratorien unter Routinebedingungen: Untersuchungen zur Fehlerkritik und Methodenkontrolle
}

Von B. Müller-Oerlinghausen

Laboratorium für Klinische Psychopharmakologie der Psychiatrischen Klinik der Freien Universität Berlin und

\section{E. Riedel}

Institut für Biochemie der Freien Universität Berlin

(Eingegangen am 8. Dezember 1977/3. Mai 1978)

Zusammenfassung: Bestimmungsverfahren von Antiepileptika unter Routinebedingungen in klinischen Laboratorien werden verglichen, und Möglichkeiten und Probleme der Methodenkontrolle und Fehlerermittlung erörtert. Für gaschromatographische und photometrische Verfahren werden Standardisierungs- und Auswerteverfahren über einen längeren Zeitraum verfolgt und ihre Genauigkeit geprüft. Empfehlungen für eine Methodenauswahl unter klinischen Bedingungen werden gegeben.

\section{Simultaneous determination of antiepileptic drugs in clinical laboratories: Problems of quality control and standardization}

Summary: Methods for determination of antiepileptic drugs in clinical laboratories are discussed with respect to precision, constant error control and economy. Experimental data for reproducibility and standardization are presented for gas chromatographic and photometric methods. Methods for choosing the most suitable procedure for any of the common analytical tasks that may arise in the clinical laboratory are recommended.

\section{Einführung}

In der „Denkschrift Epilepsie“ der Deutschen Forschungsgemeinschaft (1) wurden für den Bereich ,Medikamentöse Therapie" unter anderem als wichtige Forschungsthemen genannt:

„Vergleich und Entwicklung von Methoden und Techniken zur quantitativen Bestimmung antiepileptischer Substanzen ..., Technische Aspekte instrumenteller Methoden, Vorżüge und Nachteile von Spektrophotometrie, Dünñsçhichtchromatographie, immunologischen Verfahren und Gaschromatographie. Klinișche Anwendung der Nachweismethoden mit dem Ziel, die Bedingungen für Resorption, Metabolisièrung, . . . und Eliminierung von Antiepileptika aufzuklären sowie die Bedeutung der Serumḳonzentrationsbestimmung zur Uberwachung von Wirkung und Nebenwirkungen zu ermitteln."

Richens (2) weist auf die Bedeutung pharmakoanalytischer Therapiekontrolle in folgenden Fällen hin: ,unkontrollierte Anfälle, Anfallshäufung, mögliche Intoxika- tion, ungeklärte neurophysiologische Symptome, status epilepticus, Einfuihrung von Kombinationspräparaten, mögliche Nichteinnahme des Arzneimittels durch den Patienten, Schwangerschaften, pädiatrische antikonvulsive Therapie" (vgl. auch Schmidt l. c. (3)). Für die weite Verbreitung von Serumspiegel-Kontrollen sprechen auch die vielzähligen methodischen Einzelmitteilungen der vergangenen Jahre, deren Bibliographie nicht Gegenstand dieser Arbeit ist. Uberraschend ist allerdings, $\mathrm{daß}$ darunter kaum vergleichende pharmako-analytische Untersuchungen zu finden sind, wie sie von der „Denkschrift Epilepsie" dringend gefordert wurden.

Die zur Zeit klinisch und ambulant häufigst applizierten Antiepileptika sind Phenobarbital, Pheny toin, Carbamazepin, Primidon, Ethosuximid, Dipropylacetat, Sultiam (vgl. Riedel 1. c. (4)), die in Einzel- und Kombinationstherapien eingesetzt werden. Für eine fehlerfreie Konzentrationsbestimmung im Serum ist eine genaue Kenntnis der Medikation inklusive aller gleichzeitig applizierter Arzneimittel für das Labor wesentliche Voraussetzung. Danach richtet sich die Methodenauswahl. Im klinischen 
Labor ergibt sich in der Pharmakoanalytik aus zwei wesentlichen Gründen die Notwendigkeit, mehrere unabhängige Bestimmungsmethoden simultan zur Verfügung zu haben:

1. Eine kontinuierliche Qualitätskontrolle muß im eigenen Laboratorium möglich sein. Der Anschluß an Ringversuche oder der Bezug auf Kontrollseren gewährleistet erfahrungsgemäß noch keine ausreichende Analysensicherheit (vgl. Kupferberg \& Penry 1. c. (5)).

2. Kostenersparnis und Kapazitätserweiterung sind möglich bei einer an der Häufigkeit der einzelnen Analy senprobleme orientierten Auswahl der einzusetzenden Methode.

Zur Qualitätskontrolle sind die klinischen Laboratorien auch in der Pharmakoanalytik zweckmäßigerweise in Ringversuchen zusammengeschlossen. Solche Ringversuche dienen im wesentlichen einer besseren Vergleichbarkeit von Analysenergebnissen verschiedener Laboratorien. Zur Aufdeckung methodischer Fehler werden jedoch unabhängige Methoden benötigt. Auf dieses Problem weisen auch die Ergebnisse eines internationalen Ringversuches in der Analytik von Antiepileptika eindeutig hin (6). Im klinischen Laboratorium werden aus Gründen der Kostenersparnis und größeren Kapazität photometrische Bestimmungsmethoden stets bevorzugt, wie im Falle der Antiepileptika zum Beispiel die - auch in unseren Laboratorien - seit langer Zeit erfolgreich eingeführte Simultanbestimmung von Phenytoin und Phenobarbital nach Svensmark \& Kristensen (7). Da solche Methoden jedoch gegenüber Begleitmedikationen störanfällig sind, sollten bei Kombinationstherapien gaschromatographische oder dünnschichtchromatographische Trennverfahren eingesetzt werden. Letztere sind oft preiswert, aber dafür zeitaufwendig. Hochdruckliquidchromatographische Verfahren erwiesen sich uns bisher als noch zu störanfällig im apparativen Bereich, der Enzymimmunoassay (EMIT) als vergleichsweise zu kostenintensiv ${ }^{\mathbf{1}}$ ). Bei sachgerechter Anwendung bietet die Gaschromatographie einen hohen Grad an Anpassungsfähigkeit an das einzelne Analysenproblem und eine hohe Analysenkapazität. Bei richtiger Anwendung ermöglicht der gleichzeitige Einsatz von photometrischen und gaschromatographischen Methoden die gegenseitige, unabhängige Methodenkontrolle, die die Sicherheit für die Richtigkeit der Analysenergebnisse liefert. Aus einer Analysenzahl von mehr als 2000 Patienten-Untersuchungen haben sich die folgenden Empfehlungen für eine Methodenauswahl bei der Bestimmung von Antiepileptika ergeben.

1) Phenobarbitalbestimmungen nach dem EMIT-Verfahren waren in unseren Laboratorien z. B. in Serien von 100 Proben über 5-10 Arbeitstage bezüglich der Materialkosten 12fach teurer als gaschromatographische Bestimmungen.

\section{Material und Methoden}

Reagenzien und Arzneimittel

Dichlormethan, Chloroform, Dichlorethan, Ethanol, Phthalimid, Stigmasterin als p. a.-Qualität Merck; Chromosorb G, Gaschrom $Q$ (je 100/120 mesh), Dichlordimethylsilan, Silikon:OV-17 in GLC-Qualität der Fa. W. G. A., Düsseldorf. Phenobarbital (Luminal), Sultiam (Ospolot) stellten die Bayer-Werke als Reinsubstanz, Primidon (Mylepsin), Ethosuximid (Pyknolepsin) die Fa. ICI-Pharma, Carbamazepin (Tegretal) die Fa. Geigy AG, Pheny toin (Phenhydan) die Fa. Desitin-Werke in Reinsubstanz zur Verfügung.

\section{Apparaturen}

Photometrische Bestimmungen wurden an einem BeckmanDBG bzw. Zeiss-ELCO-Filterphotometer durchgeführt.

Gaschromatographie: Folgende Geräte wurden eingesetzt: Varian-Aerograph 1520B mit Doppel-FID, Glassäulen $90 \mathrm{~cm}$, $2 \mathrm{~mm} \phi, 3 \%$ Silik on OV-17 auf Chromosorb GAW-DMCS 100/120 mesh bzw. Gaschrom Q 100/120 mesh. - SiemensChromatograph L4000, Doppel-FID, Glassäulen $100 \mathrm{~cm}$, $2 \mathrm{~mm} \phi$, gepackt wie vorstehend (Leihgabe der DFG). - Gaschromatograph Packard-Becker 420 mit Doppel-FID, Glassäulen $180 \mathrm{~cm}, 2 \mathrm{~mm} \phi$, gepackt wie vorstehend. - Trägergas: $\mathrm{N}_{2}, 40 \mathrm{ml} / \mathrm{min}$, Brenngas: $\mathrm{H}_{2} 30 \mathrm{ml} / \mathrm{min}$, Luft $300 \mathrm{ml} / \mathrm{min}$. Elektronischer Integrator HP $3380 \mathrm{~A}$ (Hewlett-Packard).

\section{Stand ardlösungen}

Ausgangslösungen für Standardisierungen und Wiederfindungsbestimmungen waren Lösungen von $1 \mathrm{~g} / \mathrm{l}$ Phenytöin, Phenobarbital, Ethosuximid, Primidon bzw. Carbamazepin in Etha$\mathrm{nol}^{2}$ ). Als apparative interne Standardlösung der Gaschromatographie diente eine Lösung von $1 \mathrm{~g} / 1$ Stigmasterin und/oder $1 \mathrm{~g} / 1$ Phthalimid in Ethanol, für Einzelbestimmungen von Phenobarbital eine Lösung von Methylphenylethylhydantoin in Ethanol.

\section{Bestimmung der Verteilungsk oeffizienten}

Je $5 \mathrm{ml}$ von Lösungen von $10 \mathrm{mg} / 1$ Pheny toin, Phenobarbital, Primidon bzw. Ethosuximid in entsprechenden Lösungsmitteln (Dichlormethan, Chloroform, Dichlorethan) wurden mit $5 \mathrm{ml}$ wäßriger Pufferlösung (Glycinpuffer bzw. Sörensenpuffer) pH 4,0 - pH 11,0 geschüttelt. Danach wurden $2 \mathrm{ml}$ der wäßrigen Phase photometrisch gegen Leerpuffer vermessen; $2 \mathrm{ml}$ organische Phase wurden unter $\mathrm{N}_{2}$ eingedampft, mit $50 \mu \mathrm{l}$ Lösung des internen Standard aufgenommen und gaschromatographiert. Der Mittelwert beider Bestimmungen wurde in den Abbildungen 1a-c dargestellt.

\section{Extraktionsgang}

$3 \mathrm{ml}$ Serumprobe werden mit $2 \mathrm{ml}$ Pufferlösung $\mathrm{pH} \mathrm{5,0} \mathrm{und}$ $5 \mathrm{ml}$ Dichlorme than $10 \mathrm{~min}$ geschüttelt, bei $3000 \mathrm{U} / \mathrm{min}$ zentrifugiert, die organische Phase wird mit einer Injek tionsspritze von unten abgezogen. Es wird ein 2 . Mal mit $5 \mathrm{ml}$ Dichlormethan extrahiert. Die vereinigten Dichlormethan-Phasen werden mit $50 \mu$ l Ethanol aufgenommen und nach sorgfaltigem Lösen der gaschromatographischen Analyșe zugefuhrt. Beim Eindampfvorgang besonders für die Isolierung von Ethosuximid dürfen $25^{\circ} \mathrm{C}$ nicht überschritten werden.

\section{Gasverteilungschrom atograph ischer Trennungsgang}

Die gaschromatographischen Trennungen wurden mit o.g. Trennsäulen und Gasflüssen, die über ein van Deemter-Diagramm optimiert wurden (vgl. Schley et al., 1. c. (8)) bei folgenden Säulentemperaturen durchgeführt: $130^{\circ} \mathrm{C}$ zur Bestimmung von Ethosuximid mit Phthalimid als apparativem internen Standard, $220^{\circ} \mathrm{C}$ zur Bestimmung von Phenobarbital mit Methylphenylethylhydantoin, $270^{\circ} \mathrm{C}$ zur Bestimmung von

$\left.{ }^{2}\right)$ Für die Umrechnung in SI-Einheiten (umol/l) gelten folgende Multiplika toren: Pheny toin: 397; Phenobarbital: 431; Ethosuximid: 792; Primidon: 4.5.9; Carbamazepin: 424. 

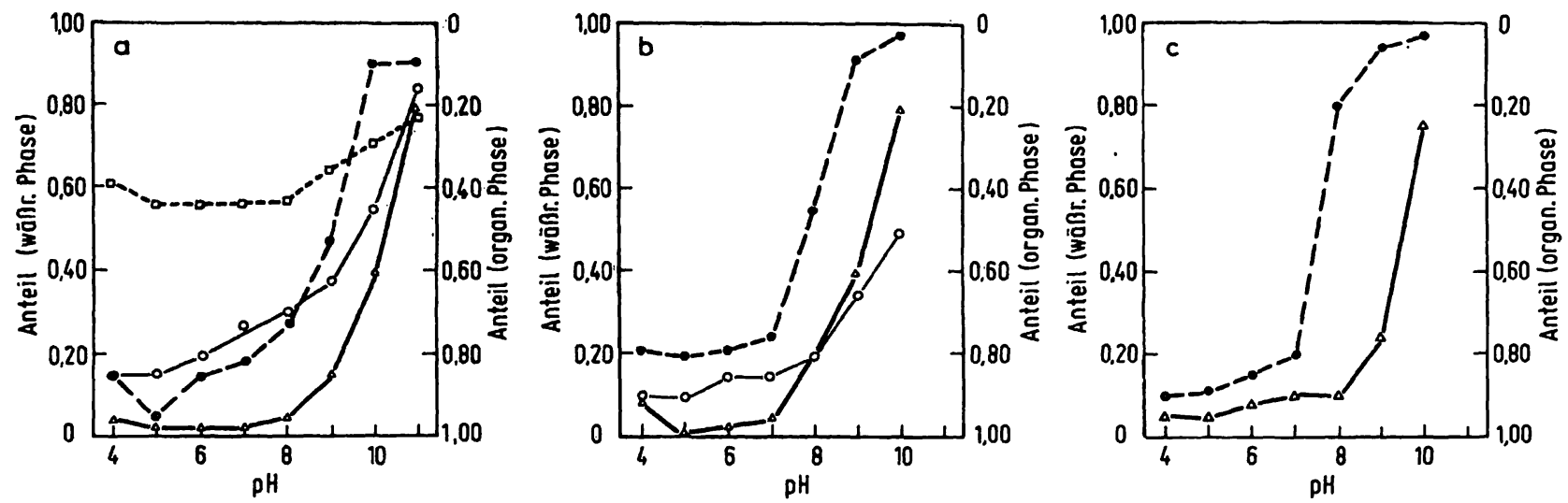

Abb. 1. Verteilungsdiagramme für Antiepileptika zwischen wäßrigen Phasen pH 4-11 und a) Dichlormethan, b) Chloroform, c) Dichlorethan.

$\bullet--$ Phenobarbital $\quad-\circ$ Ethosuximid $\Delta-\Delta$ Phenytoin $\quad 0-\square$ Primidon

Pheny toin und Carbamazepin oder Sultiam mit Stigmasterin als apparativem internen Standard. Als Temperaturprogramm bei Dreifachbestimmungen von Ethosuximid, Phenobarbital und Pheny toin nebeneinander wurde nach Injektion der Probe mit Phthalimid und Stigmasterin als internen apparativen Standards von $130-260^{\circ} \mathrm{C} \mathrm{mit} 10^{\circ} \mathrm{C} / \mathrm{min}$ geheizt und eine isotherme Stufe von 15 Minuten nachgeschaltet. Alternativ wurde nach $3 \mathrm{~min}$ isothermer Vorstufe $\left(130^{\circ} \mathrm{C}\right) \mathrm{mit} 10^{\circ} \mathrm{C} / \mathrm{min}$ auf $270^{\circ} \mathrm{C}$ geheizt und 7 Minuten isotherm nachgefahren. Zur Doppelbestimmung von Pheny toin und Phenobarbital wurde in diesem Fall Tolylphenylhydantoin als interner Standard verwendet.

\section{Ergebnisse}

\section{Extraktionstechnik}

Die photometrische Simultanbestimmung von Phenytoin und Phenobarbital nach Svensmark \& Kristensen (7) hat eine genaue pH-Einstellung der wäßrigen Serumphase und Abpufferung dieses $\mathrm{pHs}$ vor der Extraktion mit organischen Lösungsmitteln zur Voraussetzung. Fehler bei der Bestimmung nach dieser Methode beruhen häufig auf einer ungenügenden Kontrolle der $\mathrm{pH}$ Werte der Pufferlösungen. Die Antiepileptika Phenytoin, Ethosuximid, Phenobarbital und Primidon sind schwache Säuren und daher aus saurer, wäßriger Phase zu extrahieren. Auch für Extraktionen bei gaschromatographischen Bestimmungsmethoden ist auf die Einhaltung der pH-Werte der wạßßrigen Serumphasen genau zu achten. Zu starkes Ansäuern mit hochkonzentrierten Säuren ist $z u$ vermeiden, da dann bereits hydrolytische Zersetzungsreaktionen auftreten können. Wir haben zur Ermittlung der günstigsten Extraktionsbëdingungen die Verteilungskoeffizienten einiger Antiepileptika zwischen wäßrigen Lösungen von $\mathrm{pH}$ 4-11 und den Halogenalkanen Dichlormethan, Chloroform, Dichlorethan ermittelt, wobei Dichlormethan wegen seines niedrigen Verdampfungspunktes (rasches Eindampfen unter $\mathrm{N}_{2}$ möglich) ein geeignetes Extraktionsmittel zur Vorbereitung gaschromatographischer Trennung, Chloroform in der Regel das Extraktionsmittel für photometrische Bestimmungen, Dichlorethan ein häufig für nachfol- gende dünnschichtchromatographische Bestimmungen verwendetes Extraktionsmittel ist (Abb. 1a-c). Die günstigsten pH-Werte für Extraktionen liegen im Bereich pH 5-6. Mit Ausnahme von Primidon werden bei allen drei Extraktionsmitteln nach 2 maliger Extraktion bereits mehr als $96 \%$ in diesem pH-Bereich aus wäßriger Phase extrahiert. Um gute Wiederfindungsraten für Primidon zu erhalten, sind mindestens 3 aufeinanderfolgende Extraktionen durchzuführen, um etwa 80\% des Pharmakons der Detektion zuführen zu können. Inwieweit durch den oben skizzierten Extraktionsgang die Proteinbindungen des Pharmakons gelöst und/ oder die in Serumlipiden gelösten Pharmakoanteile herausgelöst werden, zeigt am Beispiel Pheny toin (mit Dichlormethan-Extraktion) Abbildung 2 an einem Vergleich von in wäßriger $\mathrm{NaCl}$-Lösung und Serumproben eingewogenen Phenytoin-Mengen. Bei der hier verwendeten Extraktionsmethode wird also die Gesamtkonzen-

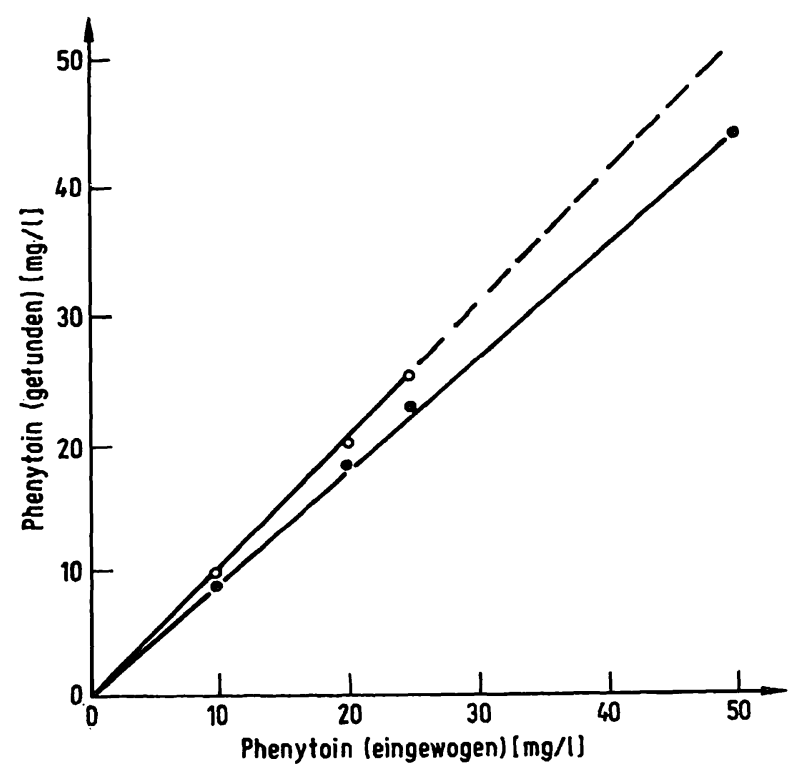

Abb. 2. Wiederfindungsrate von eingewogenem Phenytoin aus wäßriger $\mathrm{NaCl}$-Lösung $(\mathrm{o}-\mathrm{O})$ bzw. aus Humanserum $(\bullet-\bullet)$. 
tration im Serum ermittelt. Die von uns eingesetzte 2stufige Extraktionstechnik kann nur bei nachfolgender direkter Aufnahme des Eluats auf die gaschromatographische Säule empfohlen werden. Für Bestimmungen nach Kupferberg (9) mit Pharmakon-Methylierung auf der Säule mittels Trimethylaniliniumhydroxid ist eine mehrstufige Reinigung der Extrakte unerläßlich. Das bedeutet zusätzliche Substanzverluste während des Aufbereitungsganges.

\section{Gasverteilungschromatographische Trennung}

Bevor wir in unseren Laboratorien als Trennsystem 3\% OV-17/Chromosorb G (100/120) AW DMCS einsetzten, haben wir die Trenn- und Empfindlichkeitseigenschaften folgender anderer Systeme getestet: $2 \%$ Carbowax $20 \mathrm{M}$, $3 \%$ QF-1/XE 60 (8/1), 3\% XE 60, 3\% Silicon-SE 30 auf Chromosorb P-, W-, G-AW DMCS der Siebgrößen 80/100 bzw. 100/120 mesh und Gaschrom Q (100/120 mesh). Auf die Problematik des sog. ,magischen Dreiecks" der Chromatographie hat Engelhardt (10) ausführlich hingewiesen: im Kompromiß zwischen optimaler Auflösung - Analysengeschwindigkeit - Belastbarkeit der Systeme hat sich das eingesetzte Trennsystem allen anderen überlegen erwiesen. Die Frage der Belastbarkeit spielt in unserem Zusammenhang insofern eine Rolle, als verschiedene Anteile an serumeigenen Verbindungen durch die Trennung mitgeschleppt werden.

Wesentliche Voraussetzungen für reproduzierbare, quan- titative gaschromatographische Pharmakon-Bestimmungen sind

1. ständige Kontrolle des Trennsystems

(Kontrollkalibrierungen zwișchen den Analysenproben),

2. täglicher Kontrollwert einer im Leerserum eịngewogenen Substanzprobe

(tägliche Ǔberprüfung der Wiederfindungșrate).

Diese Kontrollen werden aus Kapazitätsgründen häufig zum Nachteil der Analysensicherheit minimiert. Daher weisen wir auf ihre Wichtigkeit hin.

\section{Kontrolle des Trennsystems}

Die apparative Standardisierung erfolgt durch Aufnahme von Stanḍardkurven mit Standardlösungen Pharmakon + interner Standard mit einem Standardsatz $(0,25$; 0,$50 ; 0,75 ; 1,00 \mathrm{~g} / 1$ Pharmakon + jeweils $1,00 \mathrm{~g} / 1$ interner Standard in Ethanol), der in Glasgefäßen gut verschlossen über mehrere Wochen haltbar ist. Die Auswertung der Chromatogramme ist durch Peaakflächenvergleich oder Peakhöhenvergleich möglich.

Solche Standardkurven sind unerläßlich, da bereits Kupferberg (9) darauf hinwies, daß besonders bei Peakhöhenvergleichskurven häufig keine Standardgeraden mit Verlauf durch den Nullpunkt zu erzielen sind. Wir geben daher in Abbildung $3 \mathrm{a}-\mathrm{f}$ solche Standardkurven im Peakhöhenvergleich an. Die Standardsätze müssen

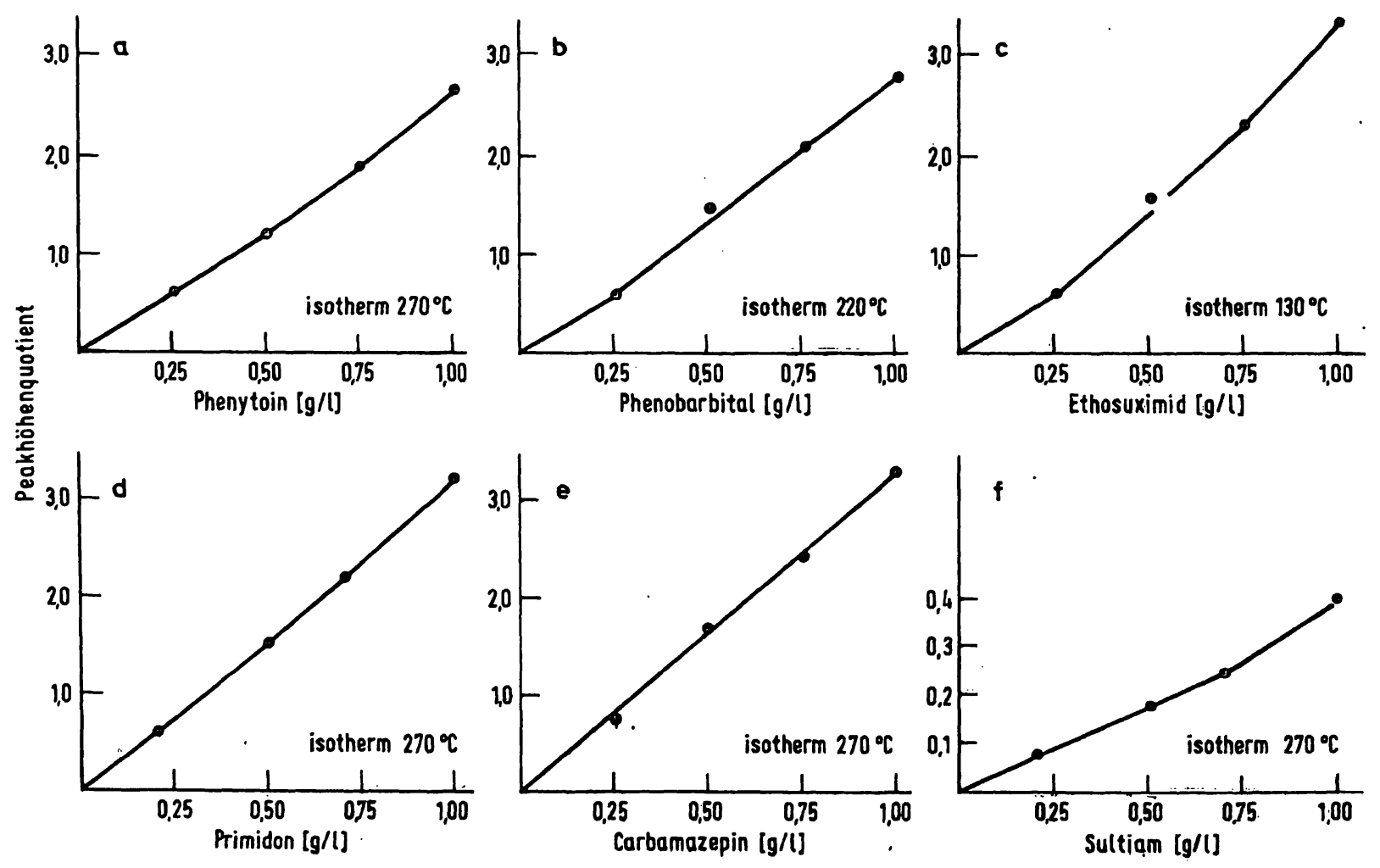

Abb. 3. Typische apparative Standardkurven bei Auswertung mit Peakhöhen (h)-Quotienten $Q=h_{\text {Substanz }} / h_{\text {Interner Standard }}$. Interner Standard zu a), d-f) Stigmasterin, zu b) Methylphenylethylhydantoin, zu c) Phthalimid, jeweils. 1 g/l Ethanol. Die Pharmaka wurden in den Lösungen der internen Standards gelöst. 


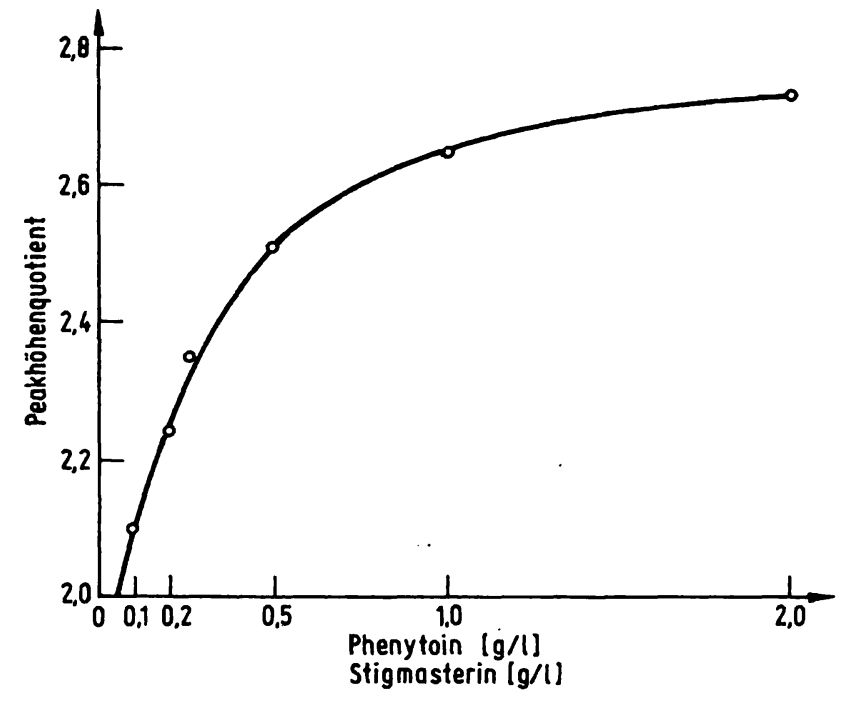

Abb. 4. Änderung des Peakhöhen-Quotienten $Q=$ Peakhöhe $_{\text {Phenytoin }} /$ Peakhöhe $_{\text {Stigmasterin }}$ mit der Verdünnung durch Lösungsmittel (Ethanol) bei gaschromatographischer Trennung (vgl. Text) Konzentrationsverhältnis Phenytoin/Stigmasterin $=1: 1$.

also in Konzentrationsbereichen zusammengesetzt werden, die dem klinisch angewandten Konzentrationsbereich des jeweiligen Pharmakons entsprechen. Für Phenytoin z. B. erzielt man nur bei einer Konzentration $>0,5 \mathrm{~g} / 1$ in Ethanol (Einspritzmenge je $1 \mu \mathrm{l}$ ) hohe Analysengenauigkeit bei dem hier angewendeten Analysenweg (Abb.4). Das ist in den Standardkurven der Bereich von $0,50-1,00 \mathrm{~g} / 1$ interner Standardlösung ( $1 \mathrm{~g} / 1$ Stigmasterin in Ethanol). Bei Injektion verdünnter Lösungen sinkt die Nachweisempfindlichkeit des Trennsystems bei gleichzeitiger erheblicher Verkleinerung des Peakhöhen-Quotienten. Auf die Bedeutung der Kontrolle und Stabilität der Injektortemperatur bei gaschromatographischen Bestimmungen wurde bereits früher hingewiesen (4). Bei Berücksichtigung dieser Faktoren haben wir die Standardkurven für Phenytoin und Ethosuximid über ein Jahr verglichen. Folgende Regressionsgeraden wurden erhalten:

für Phenytoin:

$Q=0,1796+2,93 c(r=0,987)$ und

für Ethösuximid:

$Q=0,1672+3.01 c(r=0,985)$.

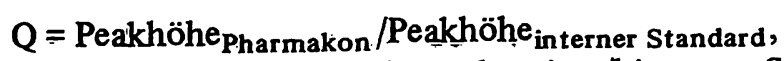

$\mathrm{c}=$ Konzentration des Pharmakón in $\mathrm{g} / \mathrm{l}$ interner Standard im geprüften Konzentrationsbereich.

Das zeugt für die Qualität der Methode. Wir sind der Uberzeugung, daß die Vernachlässigung dieser Kontrolle die Hauptursache ist für die Analysenwertabweichung bei Ringversuchen (6) sowie methodische systematische Abweichungen, wie sie z. B. von Janz \& Schmidt (11) zwischen photometrischen und gaschromatographischen Werten beobachtet wurden. In diesem Zusammenhang erschien uns eine Abschätzung des methodischen Fehlers bẻi Flächen- und Höhenver- gleichsauswertung der Gaschromatogramme wichtig und ist an den Beispielen der Phenytoin und Phenobarbital-Standardkurven (Abb. 5) veranschaulicht. Der methodische Fehler ist konzentrationsabhängig. Die Peakhöhenquotienten sind etwas größer als die Peakflächenquotienten, korrelieren aber exakt miteinander. Die Variationskoeffizienten für die einzelnen Meßwerte (Tab. 1) geben wichtige Hinweise auf die optimalen Konzentrationsverhältnisse von Substanz und zugehörigem internen Standard. Die Variationskoeffizienten bei Peakflächenauswertung liegen erwartungsgemäß niedriger als bei Peakhöhenauswertung, die Peakflächenauswertung wird aber bei ungünstigen Konzentrationsverhältnissen Substanz/Interner Standard $(<1,5)$ erheblich fehlerhafter. Diese durch Konzentrationsverhältnisse bedingten Fehler hängen in der Regel auch von der Retentionszeitdifferenz zwischen Substanz und internem Standard ab. Für die in dieser Arbeit eingesetzten internen Standards sind durchschnittliche Konzentrationsverhältnisse von Substanz: Standard von etwa 1:1 anzustreben.

In der Praxis zeigte uns ein Vergleich von 24 Phenytoin-Bestimmungen in Patientenseren, daß die elektronische Flächenintegration (Peakflächenvergleich $Q_{F}$ ) mit Computerberechnung der vorgefundenen Konzentration (ausgedruckter Wert AMT = amount) durchaus nicht geringere Fehlerabweichungen bringt als eine exakt durchgeführte Peakhöhenvergleichsauswertung $\left(\mathrm{Q}_{\mathrm{H}}\right)$ (Tab. 2).

\section{Kontrolle der Widerfindungsrate}

Recoverybestimmungen gehören zum selbstverständlichen Bestand der Analysenkontrolle. Die hier eingesetzte Methode der Standardisierung mit einem appara-

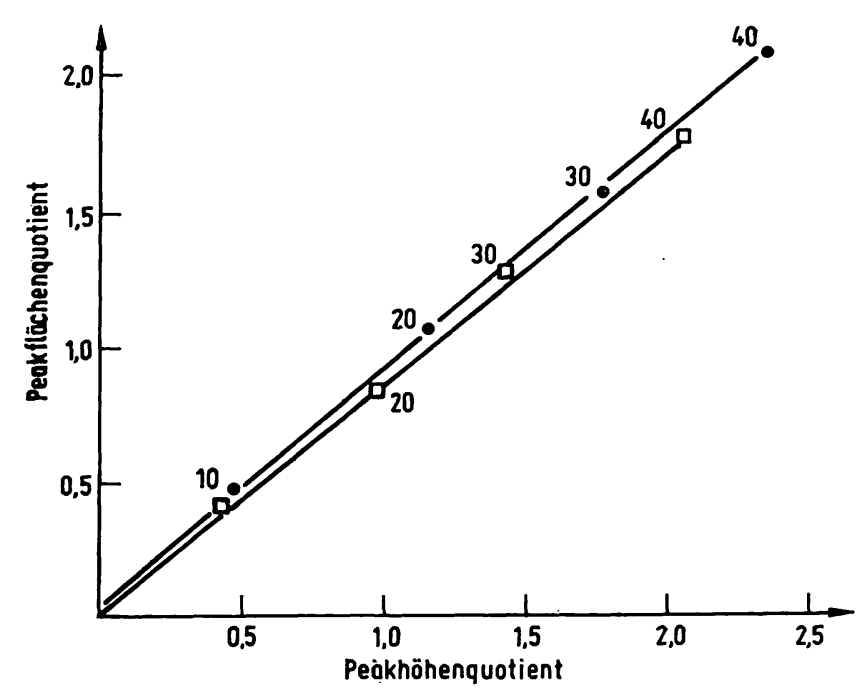

Abb. 5. Korrelation der quantitativen Auswertung von Phenytoin-(•)- und Phenobarbital-(a)-Standardkurven mit 10, $20,30,40 \mu \mathrm{g}$ Substanz gegen $20 \mu \mathrm{g}$ in ternen Standard (Stigmasterin bzw. Methylphenylethylhydantoin) mittels Peakhöhenquotient $\left(Q_{H}\right)$ bzw. Peakflächenquotient $\left(Q_{F}\right)$. 
Tab. 1. Fehlerabschätzung für Standardkurven von Phenytoin bzw. Phenobarbital bei Peakhöhen- $\left(\mathrm{Q}_{\mathrm{H}}\right)$ bzw. Peakflächenvergleichsauswertung $\left(Q_{F}\right) \cdot \bar{x}=$ Mittelwerte der Standardlösungen mit 10-40 $\mu \mathrm{g}$ Substanz und je $20 \mu \mathrm{g}$ inneren Standard.

\begin{tabular}{|c|c|c|c|c|c|c|c|}
\hline & \multirow[b]{2}{*}{$\mathbf{N}$} & \multicolumn{2}{|l|}{$\mathrm{Q}_{\mathrm{H}}$} & \multirow[b]{2}{*}{ VK (\%) } & \multicolumn{2}{|l|}{$Q_{F}$} & \multirow[b]{2}{*}{ VK (\%) } \\
\hline & & $\bar{x}$ & $s_{x}$ & & $\overline{\mathbf{x}}$ & $s_{\mathbf{x}}$ & \\
\hline $\begin{array}{l}\text { Phenot } \\
10 \mu \mathrm{g} \\
20 \mu \mathrm{g} \\
30 \mu \mathrm{g} \\
40 \mu \mathrm{g}\end{array}$ & $\begin{array}{l}17 \\
15 \\
13 \\
20\end{array}$ & $\begin{array}{l}0,433 \\
0,959 \\
1,408 \\
2,067\end{array}$ & $\begin{array}{l}0,097 \\
0,205 \\
0,298 \\
0,333\end{array}$ & $\begin{array}{l}22,4 \\
21,4 \\
21,2 \\
16,1\end{array}$ & $\begin{array}{l}0,388 \\
0,842 \\
1,309 \\
1,777\end{array}$ & $\begin{array}{l}0,061 \\
0,120 \\
0,142 \\
0,360\end{array}$ & $\begin{array}{l}15,6 \\
14,3 \\
10,8 \\
20,2\end{array}$ \\
\hline $\begin{array}{l}\text { Phenyt } \\
10 \mu \mathrm{g} \\
20 \mu \mathrm{g} \\
30 \mu \mathrm{g} \\
40 \mu \mathrm{g}\end{array}$ & $\begin{array}{l}17 \\
16 \\
12 \\
20\end{array}$ & $\begin{array}{l}0,482 \\
1,149 \\
1,762 \\
2,358\end{array}$ & $\begin{array}{l}0,112 \\
0,220 \\
0,323 \\
0,374\end{array}$ & $\begin{array}{l}23,2 \\
19,2 \\
18,3 \\
15,9\end{array}$ & $\begin{array}{l}0,493 \\
1,054 \\
1,585 \\
2,092\end{array}$ & $\begin{array}{l}0,067 \\
0,118 \\
0,190 \\
0,379\end{array}$ & $\begin{array}{l}13,6 \\
11,2 \\
11,9 \\
18,1\end{array}$ \\
\hline
\end{tabular}

Tab. 2. Fehlervergleich der Auswertung von Gaschromatogrammen im Peakhöhenvergleich $\left(Q_{H}\right)$, Peakflächenvergleich $\left(Q_{F}\right)$ bzw. im elek tronischen Auswertungsverfahren $\left(Q_{A M T}\right) N=24$ Bestimmungen von Phenytoin in Patientenseren.

\begin{tabular}{llrr}
\hline \multicolumn{4}{l}{ Pheny toin (mg/l Serum) } \\
& $\mathrm{Q}_{\mathbf{H}}$ & $\mathrm{Q}_{\mathbf{F}}$ & $\mathrm{Q}_{\text {AMT }}$ \\
\hline$\overline{\mathbf{x}}$ & 17,29 & 18,34 & 17,98 \\
$s_{\mathbf{x}}$ & 8,44 & 9,82 & 9,89 \\
$\bar{s}_{\mathbf{x}}$ & 1,72 & 2,01 & 2,02 \\
\hline
\end{tabular}

tiven internen Standard und Korrektur der Meßwerte durch aus Wiederfindungsanalysen bestimmten Korrekturfaktoren hat sich als verläßlicher erwiesen als die Eingabe von internen Standards in die Seren, deren Koextraktion einen zusätzlichen, häufig unübersehbaren Störfaktor (vom Pharmakon differierende Verhaltensweise im Trenngang) bedingt. Der Verzicht auf einen dem Serum zugefügten internen Standard ist bei Sicherung einer mehr als 90 proz. Extraktion (Totalextraktion) des Pharmakons gerechtfertigt, wie sie hier bei Phenytoin, Phenobarbital und Ethosuximid gegeben ist. Tabelle 3 gibt unsere Erfahrungswerte für die am häufigsten angewendeten Antiepileptika wieder.
Im Rahmen von über einen Zeitraum von einem Jahr verteilten photometrischen Bestimmungen von eingewogenen Phenytoin- bzw. Phenobarbital-Proben haben wir vergleichsweise auch die Standardkurve Absorption A/Konzentration c Phenytoin (g/l Serum) statistisch ausgewertet

$\left(A=A_{\text {gefunden }}-A_{\text {Leerwert }}\right)$ und folgende Regression bestimmen können:

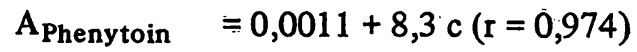

$A_{\text {Phenobarbital }}=0,0032+9,0 c(r=0,982)$

Auch hier ist die Qualität dieser Methode bei richtiger Anwendung ausgewiesen.

\section{Methodenwahl}

Bei Konzentrationsbestimmungen von Pharmaka im Serum mit nur einem Pharmakon werden wahlweise die photometrischen Tests oder isotherme GLC-Verfahren eingesetzt. Für Phenytoin und Phenobarbital sind dabei die photometrische Svensmark=Methode, für Carbamazepin die photometrische Methode nach Herrmann (vgl. auch Diehl et al., 1. c. 12) routinebewährte, kostengünstige und wenig zeitaufwendige Wege. Bei Mehrfachapplikation von Antiepileptika sind gaschromatographi-

Tab. 3. Wiederfindungsraten und Korrekturfaktoren für Konzentrationsbestimmungen von Antiepileptika im Serum.

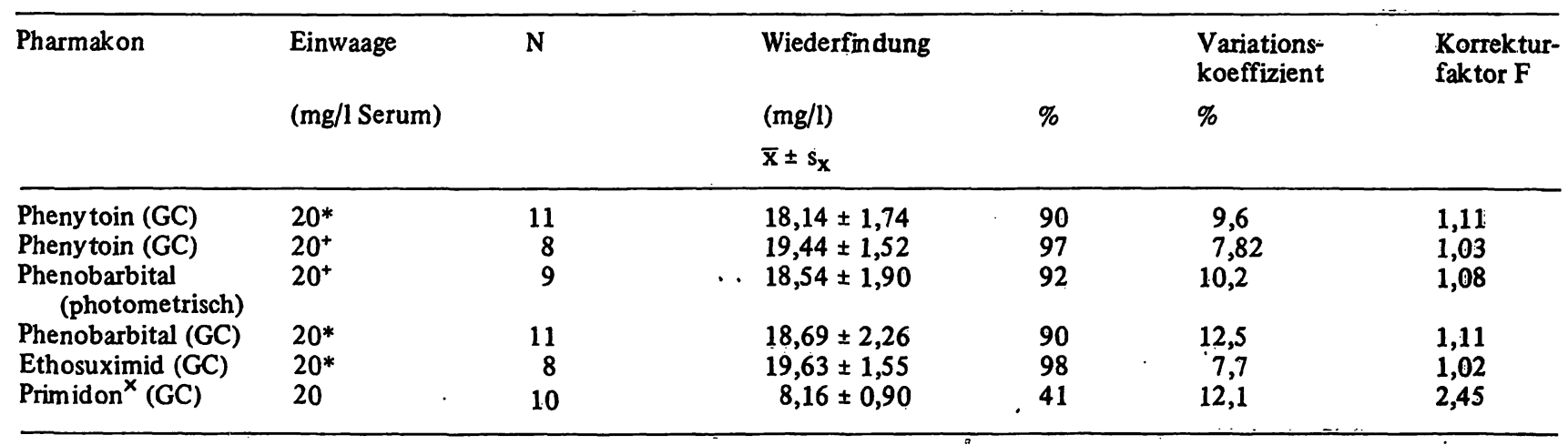

$x$ bei nur zweimaliger Extraktion

* lyophilisiertes Humanserum (Leder-Norm U), laboreigene Einwaage

+ toxikologisches Kontrollserum: Toxicology Control (Humañ) Drugs A (Lederle) 


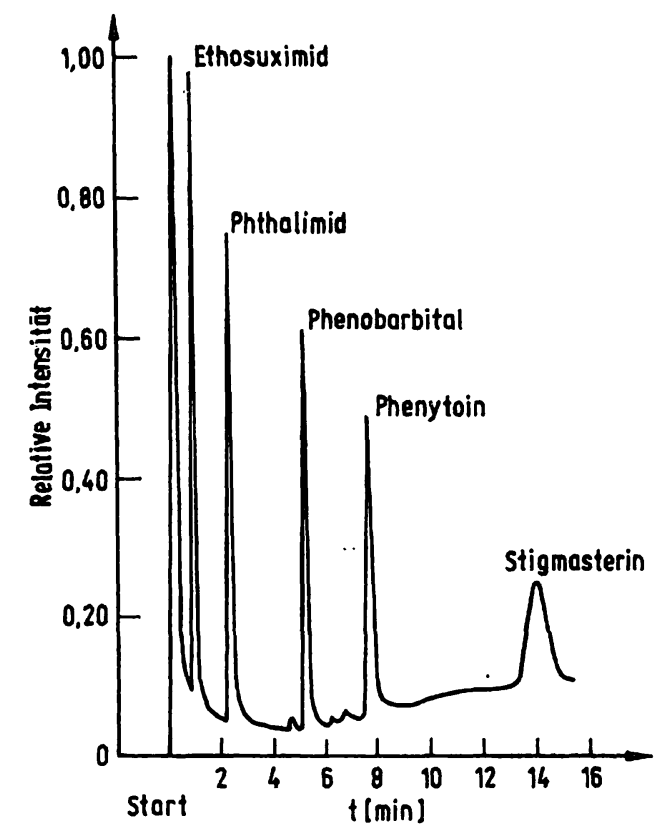

Abb. 6. Trennung von Ethosuximid, Phenobarbital und Phenytoin im direkten Temperaturprogramm-Verfahren mit Phthalimid und Stigmasterin als interne Standards. Säule: Chromosorb G AW DMCS $100 / 120$ - 3\% OV-17 $(\mathrm{L}=90 \mathrm{~cm})$, T-Programm $130-260^{\circ} \mathrm{C}, 10^{\circ} \mathrm{C} / \mathrm{min}$. Schreiber: $0,5 \mathrm{~cm} / \mathrm{min}$, Temperaturen: Verdampfer $270^{\circ} \mathrm{C}$, Detektor $300^{\circ} \mathrm{C}$ (FID).

sche Trennmethoden auf ihre Rentabilität und Kapazität zu prüfen. Temperaturprogrammverfahren, die sich z.B. bei kombinierter Gabe von Phenytoin/Phenobarbital/ Ethosuximid mit Phthalimid und Stigmasterin als apparativen internen Standard anbieten (vgl. Abb. 6), erweisen sich häufig als zeitaufwendiger als die stufenweise isotherme Bestimmung der einzelnen Komponenten. Einen Zeitvergleich der Kapazität in unserem gaschromatographischen Verfahren zeigt Abbildung 7, Beispiele für isotherme Pharmakonbestimmungen (Phenytoin, Phenobarbital, Ethosuximid) Abbildung 8. Die Reproduzierbarkeit der Peakhöhenquotienten bei isothermen Messungen ist erheblich größer als bei Temperaturprogrammen.
In unserer Routinepraxis hat es sich als empfehlenswert erwiesen, die Bestimmungen in isothermen Stufen durchzuführen. Ein Kostenvergleich der Me thoden zeigt übrigens (Tab. 4), daß insbesondere bei Mehrfachmedikation die GLC-Methoden schon aus Rentabilitätsgründen $z$. Zt. unentbehrlich sind, während z. B. der Enzymimmunoassay (EMIT) bei Kombinationsproben unverhältnismäßig teuer wird. Der EMIT-Test ist dagegen durchaus bei Einzelmedikationen und kleinerer Probenzahl als Methode für Routinebestimmungen in Betracht zu ziehen (14).

\section{Diskussion und Methodenvergleich}

In einer früheren Untersuchung haben wir an einer Reihe von Seren Phenytoin im klinisch angewandten Konzentrationsbereich bei ausgewählten Patienten mit Pheny toin-Medikation photometrisch und gaschromatographisch vergleichend bestimmt (4). Wir erhielten damals eine Regression $P=+0,90+0,976 \mathrm{G}(\mathrm{P}=$ Photometriewert, $\mathrm{G}=\mathrm{GLC}$-Wert, $\mathrm{N}=80$, Korrelationskoeffizient $\mathrm{r}=0,92$ ). Janz \& Schmidt (11) berichteten über einen ähnlichen Vergleich und erzielten eine Regression $P=7,7+0,916 \mathrm{G} ; r=0,7552$. Wir haben unsere vergleichenden Kontrollen fortgesetzt und über einen zweijährigen Zeitraum folgende Regression erhalten: $P=+1,428+0,905 \mathrm{G}$ mit einem Korrelationskoeffizienten $r=0,87$. Diese Werte wurden in zwei verschiedenen Laboratorien von verschiedenen Mitarbeitern zu unterschiedlichen Zeiten durchgeführt, d. h. unter uneinheitlichen Bedingungen. Wir können heute die Ergebnisse von Janz \& Schmidt (11) nicht bestätigen, daß GLCWerte systematisch von photometrisch ermittelten Werten abweichen. Bei methodisch bedingten Diskrepanzen müssen solche Abweichungen besonders auf folgende Ursachen hin überprüft werden:

a) ob in den Arbeitsablauf systematische Fehlermöglichkeiten eingegangen sind oder die Methoden unzureichend optimiert sind;

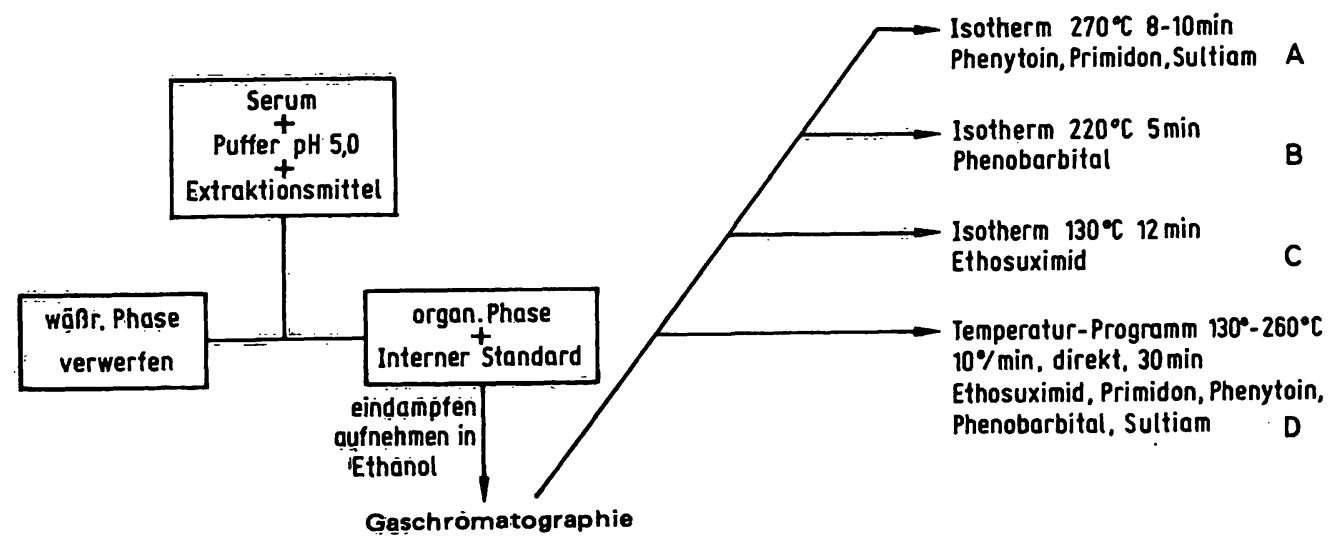

Abb. 7. Planungsschema für gaschromatographische Pharmakon-Bestimmungen. 

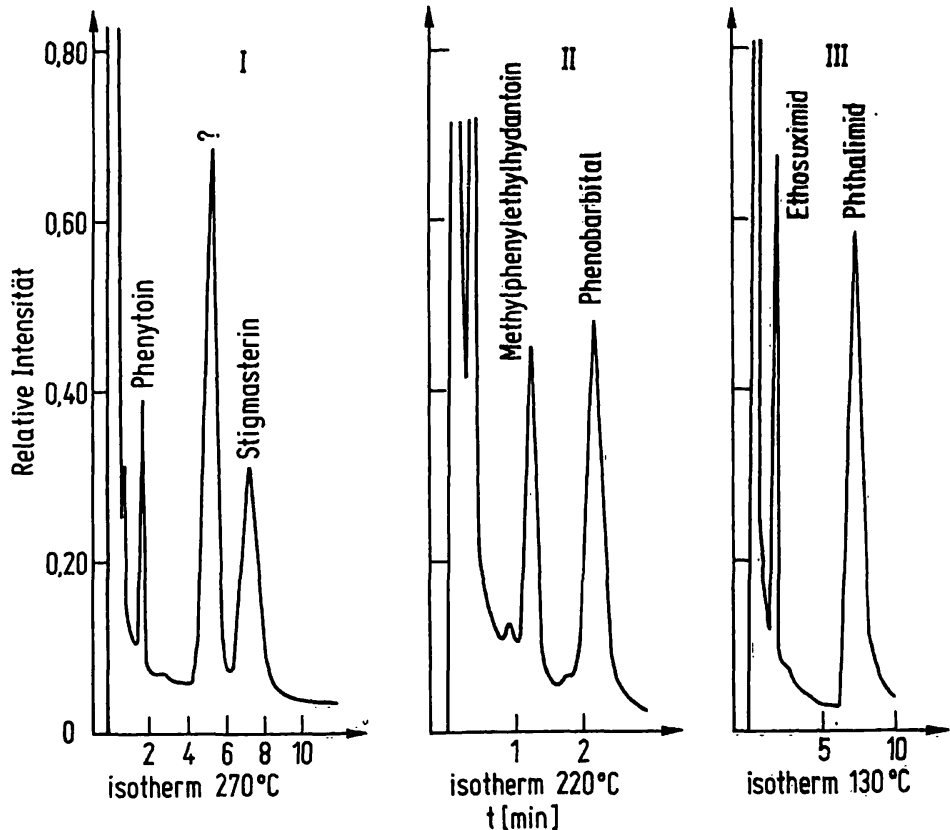

Abb. 8. Gaschromatographische Bestimmung von Antiepileptika im isothermen Verfahren.

I Patientenserum mit Phenytoin (neben Phenobarbital), Interferenz $=$ Cholesterin

II Patientenserum mit Phenobarbital (neben Phenytoin)

III Patientenserum mit Ethosuximid (neben Phenobarbital und Phenytoin).

Tab. 4. Kosten je Probe (Doppelbestimmung) unter Berücksichtigung der Kostenarten E (Einmalmaterial pro Probe und Serie), $\mathrm{R}$ (Reagenzienkosten pro Probe und Serie) und $\mathrm{P}$ (Personalkosten) in Anlehnung an den Kostenartenschlüssel von Haeckel (13) ohne Geräteamortisation. (DM pro Blutprobe im Juli 1977.)

EMIT wurde mit einem Gilford-Spektrophotometer in Verbindung mit einem HP 9825-Rechner und einem speziellen Programm durchgeführt, das schneller und komfortabler als das kommerzielle Gilford-Tectronix-Programm ist.

\begin{tabular}{llllll}
\hline Medikament & Photometrie & GLC & \multicolumn{2}{c}{ EMIT } \\
& $\begin{array}{l}6 \\
\text { Proben } \\
\text { tgl. }\end{array}$ & $\begin{array}{l}20 \\
\text { Proben } \\
\text { tgl. }\end{array}$ & $\begin{array}{l}\text { Proben } \\
\text { tgl. }\end{array}$ & $\begin{array}{l}10 \\
\text { Proben } \\
\text { tgl. }\end{array}$ & $\begin{array}{l}\text { 20 } \\
\text { Proben } \\
\text { tgl. }\end{array}$ \\
\hline Carbamazepin & $23,-$ & 6,67 & - & 31,80 & $25,-$ \\
Pheny toin & $29,-$ & 9,05 & $23,-$ & 32,67 & 23,68 \\
Ethosuximid & - & - & $23,-$ & 31,80 & $25,-$ \\
Pheny toin + Phenobarbital & $32,-$ & $10,-$ & $23,-$ & 65,34 & 47,36 \\
Phenytoin + Phenobarbital + Ethosuximid & - & - & $23,-$ & 97,14 & 72,36 \\
\hline
\end{tabular}

Tab. 5. Schema zur Methodenwahl: $1=$ Photometrie nach Svensmark \& Kristensen (7), $2=$ EMIT, $3=$ Photometrie nach Herrmann, vgl. Diehl et al. (12), 4 = GLC (A-D; vgl. Abb. 7) mit $100 \mathrm{~cm}$ bzw. $200 \mathrm{~cm}$ langer Säule.

\begin{tabular}{|c|c|c|c|c|c|c|}
\hline \multirow[t]{2}{*}{ Medikation } & \multicolumn{2}{|c|}{ Analysenmethode für } & \multirow[b]{2}{*}{$\begin{array}{l}\text { Carbama- } \\
\text { zepin }\end{array}$} & \multirow[b]{2}{*}{ Primidon } & \multirow[b]{2}{*}{$\begin{array}{l}\text { Etho- } \\
\text { suximid }\end{array}$} & \multirow[b]{2}{*}{ Sultiam } \\
\hline & Pheny toin & $\begin{array}{l}\text { Pheno- } \\
\text { barbital }\end{array}$ & & & & \\
\hline $\begin{array}{l}\text { Pheny toin } \\
\text { Phenobarbital } \\
\text { Carbamazepin } \\
\text { Ethosuximid } \\
\text { Primid on } \\
\text { Sultiam } \\
\text { Phenyt toin/Phenobarbital } \\
\text { Pheny toin/Carbamazepin } \\
\text { Pheny toin/Primidon } \\
\text { Pheny toin/Ethosuximid/Sultiam } \\
\text { Phenobarbital/Carbamazepin } \\
\text { Phenobarbital/Ethosuximid } \\
\text { Pheny toin/Phenobarbital/Primidon } \\
\text { Pheny toin/Phenobarbital/Carbamazepin } \\
\text { Primidon/(Phenobarbital)/Ethosuximid } \\
\text { Carbamazepin/Ethosuximid } \\
\text { Phenytoin/Primidon }\end{array}$ & $\begin{array}{l}1 \\
1 \\
4 A / 200 \\
4 A / 100\end{array}$ & $\begin{array}{l}4 B / 100 \\
1 \\
4 B / 100 \\
1 \\
4 B / 100 \\
4 B / 100 \\
1 \\
4 B / 100\end{array}$ & $\begin{array}{l}3 \\
3 \\
3 \\
4 A / 100\end{array}$ & $\begin{array}{l}4 A / 200 \\
4 A / 100 \\
4 A / 200\end{array}$ & $\begin{array}{l}4 C / 100 \\
4 C / 100\end{array}$ & $4 \mathrm{~A} / 100$ \\
\hline
\end{tabular}


b) ob auf dem Anforderungsformular der Klinik Begleitmedikationen, die die Methode stören, nicht eingegeben oder übersehen worden sind.

Im Laufe der Jahre hat sich in unseren Laboratorien ein Methodenauswahlschema (Tab. 5) ergeben, für das sowohl methodische, organisatorische wie finanzielle Faktoren entscheidend waren.

\section{Literatur}

1. Janz, D. (1973), Denkschrift Epilepsie. H. Boldt KG, Boppard.

2. Richens, A. (1974), Proc. Roy. Med. 67, 1227.

3. Schmidt, D. (1977), Nervenarzt 48, 183-196.

4. Riedel, E. (1975), In: Antiepileptische Langzeitmedikation (Helmchen, H., ed.), S. Karger, Basel, S. 1-14.

5. Kupferberg, H. J. \& Penry, J. K. (1975), In: Clinical pharmacology of antiepileptic drugs (Schneider, H. Janz, D. Gardner-Thorpe, C., Meinardi, H. \& Sherwin, A. L., ed.), Springer, Berlin, S. 304-305.

6. Richens, A. (1975), In: Clinical pharmacology of antiepileptic drugs. (Schneider, H., Janz, D., Gardner-Thorpe, C., Meinardi, H. \& Sherwin, A. L., ed.): Springer, Berlin, S. 293-302.

\section{Danksagung}

An den analytischen Arbeiten waren Frau $H$. Horak-Schröder, Frau I. Dellé, Herr H. Bayer (Berlin) und Frau M. Stadler (Bielefeld/Bethel) beteiligt. Wir danken ihnen sowie der Deutschen Forschungsgemeinschaft für sachliche und personelle Unterstützung (He 916/1 und Ri 191/2). Den Firmen Bayer AG, ICI-Pharma und Desitin danken wir für die Úberlassung von Arzneimittelwirkstoffen.

7. Svensmark, O. \& Kristensen, P. (1963), J. Lab. Clin. Med. 61, 501-507.

8. Schley, J., Riedel, E. \& Müller-Oerlinghausen, B. (1978), J. Clin. Chem. Clin. Biochem. 16, 307-311.

9. Kupferberg, H. J. (1970), Clin. Chim. Acta 29, 283-288.

10. Engelhardt, H. (1975), Hochd ruck flüssigchromatographie. Springer, Berlin, S. 528.

11. Janz, D. \& Schmidt, D. (1974), J. Neurol. 207, 109-116.

12. Diehl, L. W., Müller-Oerlinghausen, B. \& Riedel, E. (1976), Int. J. Clin. Pharmacol. 14, 144-148.

13. Haeckel, R. (1976), Rationalisierung des medizinischen Laboratoriums. Giebscher-Verlag, Darmstad t, S. 125-132.

14. Oellerich, M., Külpmann, W. R., Haeckel, R. \& Heyer, R. (1977), J. Clin. Chem. Clin. Biochem. 15, 353-358.

Prof. Dr. E. Riedel Institut für Biochemie der Freien Universität Berlin Limonenstraße 7 D-1000 Berlin 45 
. 